hep-th/yymmnnn

\title{
A UV completion of scalar electrodynamics
}

\author{
Pei-Ming Ho, ${ }^{a}$ Xue-Yan $\operatorname{Lin}^{b}$, \\ ${ }^{a}$ Department of Physics, Center for Theoretical Sciences \\ and Leung Center for Cosmology and Particle Astrophysics, \\ National Taiwan University, Taipei 10617, Taiwan, R.O.C. \\ ${ }^{b}$ Faculty of Law, University of Amsterdam, \\ Oudemanhuispoort 4-6 1012 CN Amsterdam, the Netherlands. \\ pmho@phys.ntu.edu.tw \\ xueyan.lin@msa.hinet.net
}

\begin{abstract}
In previous works, we constructed UV-finite and unitary scalar field theories with an infinite spectrum of propagating modes for arbitrary polynomial interactions. In this paper, we introduce infinitely many massive vector fields into a $U(1)$ gauge theory to construct a theory with UV-finiteness and unitarity.
\end{abstract}




\section{Introduction}

UV divergences in quantum field theories can be regularized by introducing higher derivatives in the kinetic term so that the propagator approaches faster to 0 than $1 / k^{2}$ at large momenta $k$. Although this is usually done at the cost of unitarity, in Ref. [1], a UV-finite $\phi^{4}$ theory in four dimensions with a higher derivative correction to the propagator of the form

$$
f\left(k^{2}\right)=\sum_{n=0}^{\infty} \frac{c_{n}}{k^{2}+m_{n}^{2}} \quad\left(c_{n}>0 \quad \forall n\right)
$$

is proved to preserve unitarity. Due to the condition $c_{n}>0$, Cutkosky's rules [3] ensure purturbative unitarity for generic Feynman diagrams.

While there are an infinite number of poles at $k^{2}=-m_{n}^{2}$ in the propagator, this theory is also equivalent to a theory with an infinite number of scalar fields with masses $m_{n}$. If $m_{n}^{2} \gg m_{0}^{2}$ for all $n>0$, the low energy behavior of this theory is approximated by an ordinary scalar field theory with a single scalar field with mass $m_{0}$. By fine-tuning the masses $m_{n}$ of the high-energy modes and the coefficients $c_{n}$, one can construct scalar field theories with arbitrary polynomial interactions in arbitrary even dimensions that are UV finite and unitary [2].

The purpose of this paper is to extend the tricks used to construct UV-finite and unitary scalar field theories to Abelian gauge theories. We start by giving a comprehensive overview of our UV-finite and unitary scalar field theories in this section. Several new insights beyond

earlier works $[1,2]$ are included. In the next section, we show how the addition of an infinite tower of fine-tuned spectrum of massive vector fields can result in UV-finiteness for a $U(1)$ gauge theory while keeping the unitarity intact. We make a few brief comments in the last section.

According to [1], in order to avoid UV divergence in the four dimensional $\phi^{4}$ theory, the following conditions are sufficient:

$$
\begin{aligned}
& \sum_{n=0}^{\infty} c_{n} m_{n}^{2}=0 \\
& \sum_{n=0}^{\infty} c_{n}=0 .
\end{aligned}
$$

To satisfy these conditions in which an infinite sum of positive numbers vanishes, analytic continuation is applied. A famous example of analytic continuation often used in string theory textbooks is the evaluation of the infinite sum

$$
\sum_{n=1}^{\infty} n \text {. }
$$


One first defines the Riemann-Zeta function by a converging series

$$
\zeta(s)=\sum_{n=1}^{\infty} \frac{1}{n^{s}} \quad(\Re(s)>1),
$$

which analytically continues to $s=-1$ so that

$$
\sum_{n=1}^{\infty} n \rightarrow \zeta(-1)=-\frac{1}{12}
$$

and the sum is identified with a negative number. The physical reason for analytic continuation can be understood as the following [2]. Due to the use of certain computational techniques or one's choice of formulation, the validity of some mathematical expressions may be restricted, but often the corresponding physical quantities can be well-defined with a larger range of validity. Relying on the analyticity of the physical problem, analytic continuation allows us to retrieve the full range of validity of our results, even though the validity of derivations is more restricted.

A closely related theory of fermions was considered earlier by Itzhaki [4]. In his theory, there are an infinite number of fermionic fields with a four-point interaction in four dimensions. The constraint on fermion masses is of the form

$$
\sum_{n} c_{n} m_{n}^{r}=0, \quad r=0,1,2,3
$$

In Ref. [2], we generalized his idea ${ }^{1}$ to $\phi^{n}$ theories in arbitrary even space-time dimensions. We adopted the same type of propagators and found that for any given $n$ and even space-time dimension $d$, we only need the conditions

$$
\sum_{n} c_{n} m_{n}^{2 r}=0 \quad \text { for } \quad r=0,1, \cdots, \frac{d-2}{2} .
$$

Remarkably this condition is independent of $n$. That is, the same propagator suits all interactions. As in [1], a set of fine tuned parameters is required for analytic continuation to be applied. The following example was given in [2]. For arbitrary $d$, let

$$
\begin{aligned}
c_{n}= & {\left[1+x_{1}(n+1)+x_{2}(n+2)(n+1)+\cdots\right.} \\
& \left.+x_{d / 2}\left(n+\frac{d}{2}\right)\left(n+\frac{d}{2}-1\right) \cdots(n+1)\right] e^{z n} \quad\left(n \geq 0, \quad x_{i} \geq 0\right), \\
m_{n}^{2}= & e^{a n} .
\end{aligned}
$$

We will set $z$ and $a$ to be positive, and we can always choose the coefficients $x_{i}$ 's such that all the conditions in (7) are satisfied. Here is how this works. Denote $\rho \equiv e^{z+a r}$ for convenience.

\footnotetext{
${ }^{1}$ Our treatment of the analytic continuation is slightly different from that of Itzhaki in Ref. [4].
} 
First assuming $\rho<1$ and then applying analytic continuation to reach the region $\rho>1$, we obtain the sum $\sum c_{n} m_{n}^{2 r}$ in the form

$$
\begin{aligned}
\sum_{n=0}^{\infty} c_{n} m_{n}^{2 r} & =\frac{1}{1-\rho}+x_{1} \frac{d}{d \rho}\left(\frac{1}{1-\rho}\right)+x_{2} \frac{d^{2}}{d \rho^{2}}\left(\frac{1}{1-\rho}\right)+\cdots x_{d / 2} \frac{d^{\frac{d}{2}}}{d \rho^{\frac{d}{2}}}\left(\frac{1}{1-\rho}\right) \\
& =\frac{1}{\xi}+\frac{x_{1}}{\xi^{2}}+\frac{x_{2}}{\xi^{3}}+\cdots \frac{x_{d / 2}}{\xi^{d / 2+1}} \equiv h(\xi),
\end{aligned}
$$

where $\xi \equiv \frac{1}{1-\rho}$, which is negative definite when $\rho>1$. By this method, we have sufficient parameters $\left\{x_{1}, x_{2} \cdots x_{d / 2}\right\}$ to fix the roots of $\xi$ at desired positions,

$$
-\left|\xi_{r}\right|=(1-\rho)^{-1}=(1-\exp (z+a r))^{-1}
$$

for $r=0,1 \cdots \frac{d-2}{2}$. These roots are all negative for $z, a>0$. We can find corresponding $x_{i}$ 's by simply comparing the coefficients with the following equation

$$
\xi^{d / 2+1} h(\xi)=c\left(\xi+\left|\xi_{1}\right|\right)\left(\xi+\left|\xi_{2}\right|\right) \cdots\left(\xi+\left|\xi_{d / 2}\right|\right),
$$

where $c$ is an arbitrary real positive number. Apparently all $x_{i}$ 's are positive because no negative coefficients appear in (11). This satisfies the condition in (1) and prevents the violation of unitarity.

In the previous work [2] we checked that the higher derivative theory with the propagator (1) can be equivalently described as a theory with ordinary kinetic terms but infinitely many fields of masses $m_{n}$ with couplings dictated by the coefficients $c_{n}$.

There is an alternative interpretation of these theories that was not discussed in earlier works. If we expand the propagator in (1)

$$
f\left(k^{2}\right)=\sum_{n} \frac{c_{n}}{k^{2}+m_{n}^{2}}=\frac{\sum_{n} c_{n}}{k^{2}}-\frac{\sum_{n} c_{n} m_{n}^{2}}{k^{4}}+\frac{\sum_{n} c_{n} m_{n}^{4}}{k^{6}}-\cdots,
$$

with the condition (2) satisfied, the first two terms in the expansion vanish. Then we sum over the remaining terms and get

$$
f\left(k^{2}\right)=\sum_{n} \frac{c_{n} m_{n}^{2}}{k^{4}}\left(\frac{m_{n}^{2}}{k^{2}}-\frac{m_{n}^{4}}{k^{4}}+\cdots\right)=\sum_{n} \frac{c_{n} m_{n}^{4}}{k^{4}\left(k^{2}+m_{n}^{2}\right)} .
$$

Defining a theory by this propagator is another approach to define the perturbation theory. It appears as if every particle in the spectrum is allowed to move at the speed of light because the $k^{2}=0$ state satisfies their equation of motion. However, we will see below that the massless excitation is not physical.

Using this propagator (13) to calculate the one-loop diagram for $\phi^{4}$ in 4 dimensions, we 


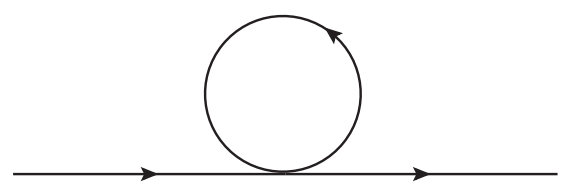

Fig 1.

get

$$
\begin{aligned}
\mathcal{M} & \propto \sum_{n} \int d^{4} l \frac{c_{n} m_{n}^{4}}{l^{4}\left(l^{2}+m_{n}^{2}\right)} \\
& \propto \sum_{n} c_{n} m_{n}^{2}\left[\log 1+\log \left(\frac{0+m_{n}^{2}}{0}\right)\right] \\
& =\sum_{n} c_{n} m_{n}^{2} \log m_{n}^{2}-\sum_{n} c_{n} m_{n}^{2} \log 0,
\end{aligned}
$$

with a potential infrared divergence in the second term. The IR divergence in fact does not exist because the coefficient $\sum_{n} c_{n} m_{n}^{2}$ vanishes as a result of the condition (2). Therefore, the one-loop diagram is finite

$$
\mathcal{M} \propto \sum_{n} c_{n} m_{n}^{2} \log m_{n}^{2}
$$

The absence of IR divergence to be cancelled by soft massless particles implies that there is no genuine massless excitation. The conditions (2) remove these massless propagating modes by destructive interference among all particles $\phi_{n} .{ }^{2}$ This suggests that the massless mode does not propagate. The absence of the massless propagating mode is crucial because the propagator $1 / k^{4}$ violates unitarity.

Another way to see that $k^{2}=0$ is not a propagating mode is to check that the propagator (13) does not diverge at $k^{2}=0$ because of (2). We rewrite the propagator (13) as a Laurent expansion of $k^{2}$,

$$
\sum_{n} \frac{c_{n} m_{n}^{4}}{k^{4}\left(k^{2}+m_{n}^{2}\right)}=\frac{\sum_{n} c_{n} m_{n}^{2}}{k^{4}}-\frac{\sum_{n} c_{n}}{k^{2}}+\sum_{n} \frac{c_{n}}{m_{n}^{2}}-\sum_{n} \frac{c_{n} k^{2}}{m_{n}^{4}}+\cdots,
$$

and find that it is actually a Taylor expansion because the $1 / k^{4}$ and $1 / k^{2}$ terms both vanish.

Many features of the theory can be explained by the propagator (1). At high energy, the propagator behave as $1 / k^{6}$. Therefore, the superficial divergence for a Feynman diagram with $L$ loops and $I$ internal lines in 4 dimensions is of the order

$$
D=4 L-6 I .
$$

Because we always have $L \leq I$ regardless of the details of interaction, the same conditions (2) for $\phi^{4}$ theory is sufficient to guarantee UV finiteness for all $\phi^{n}$ theories in 4 dimensions.

\footnotetext{
${ }^{2}$ Here "massless" excitations does not refer to the propagating mode at $k^{2}=m_{0}^{2}$ if $m_{0}$ happens to be 0 .
} 
In general $d$ dimensions, $1 / k^{6}$ at high energy does not guarantee $D<0$, more conditions are required. In (12), the first $s+1$ terms vanish if we impose the conditions

$$
\sum_{n} c_{n}=0, \quad \sum_{n} c_{n} m_{n}^{2}=0, \cdots, \quad \sum_{n} c_{n} m_{n}^{2 s}=0
$$

The propagator then behaves as $1 / k^{4+2 s}$ at high energy. To guarantee UV finiteness, we need the degree of superficial divergence to be negative, that is,

$$
D=d L-(4+2 s) I<0
$$

Since $L \leq I$, the condition $D<0$ is implied by

$$
s>\frac{d-4}{2} .
$$

Therefore, in order for the conditions (18) to guarantee UV-finiteness, the smallest possible value of $s$ is

$$
s_{\text {min }}=\frac{d-2}{2} .
$$

That is, the conditions (7) ensure UV-finiteness in $d$ dimensions.

\section{UV-finite and unitary scalar QED}

\subsection{Lagrangian}

Our ansatz for the Lagrangian of a UV-finite and unitary $U(1)$ gauge theory is

$$
\begin{aligned}
\mathcal{L} & =\sum_{n} \frac{-1}{c_{n}}\left\{\left[\left(\partial^{\mu}-i e_{n} \sum_{a=0}^{\infty} A_{a}^{\mu}\right) \phi_{n}\right]^{\dagger}\left[\left(\partial_{\mu}-i e_{n} \sum_{a=0}^{\infty} A_{a, \mu}\right) \phi_{n}\right]+m_{n}^{2} \phi_{n}^{\dagger} \phi_{n}\right\} \\
& -\sum_{a} \frac{1}{b_{a}}\left(\frac{1}{4} F_{a}^{\mu \nu} F_{a, \mu \nu}+\frac{1}{2} M_{a}^{2} A_{a}^{\mu} A_{a, \mu}\right)-V\left(\sum_{n} \phi_{n}^{\dagger} \phi_{n}\right) .
\end{aligned}
$$

We will assume the absence of scalar self interactions, that is, $V=0$. In Sec. 3 we will comment that if $V \neq 0$, UV divergence can not be avoided. As in ordinary scalar QED, this theory contains two types of fields: the scalar fields $\phi_{n}$ and the vector fields $A_{a, \mu}$. One of the spin-one particles $A_{0, \mu}$ is massless $\left(M_{0}^{2}=0\right)$ and can be recognized as the photon. The gauge transformation rules of the fields are given by

$$
\begin{gathered}
\phi_{n}(x) \rightarrow e^{-i e_{n} \Lambda(x)} \phi_{n}(x), \\
A_{0, \mu}(x) \rightarrow A_{0, \mu}(x)-\partial_{\mu} \Lambda(x), \\
A_{a, \mu}(x) \rightarrow A_{a, \mu}(x), \quad n \geq 1 .
\end{gathered}
$$


Both considerations on unitarity and UV-divergence are centered around loop diagrams. In the following we will focus on loop diagrams composed of the interaction vertices

$$
e_{n} \phi_{n}^{\dagger} \partial_{\mu} \phi_{n}\left(\sum_{a} A_{a}^{\mu}\right), \quad e_{n} \partial_{\mu} \phi_{n}^{\dagger} \phi_{n}\left(\sum_{a} A_{a}^{\mu}\right), \quad e_{n}^{2} \phi_{n}^{\dagger} \phi_{n}\left(\sum_{a} A_{a}^{\mu}\right)\left(\sum_{b} A_{b, \mu}\right) .
$$

See Fig. 2(a) and Fig. 2(b). These vertices do not $\operatorname{mix} \phi_{m}$ with $\phi_{n}$ for $m \neq n$. The transformations

$$
\phi_{n} \rightarrow e^{i \theta_{n}} \phi_{n}
$$

are global symmetries of the theory, unless the phases $\theta_{n}$ are correlated by $\theta_{n} \propto e_{n}$. The particle number of each scalar $\phi_{n}$ is conserved. Contrary to the scalar legs, the four point vertex $\phi \phi A A$ does not restrict the two vector particles to have the same index, as shown in Fig. 2(b). As a result, all vector internal lines are superpositions of all vector fields $A_{a, \mu}$.

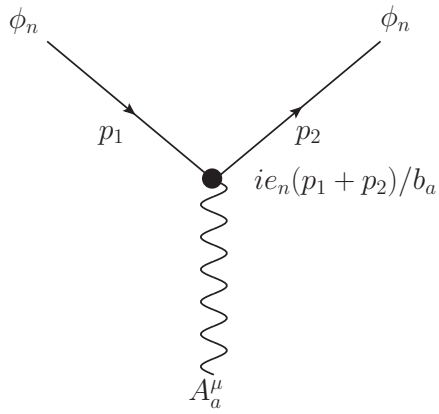

(a)

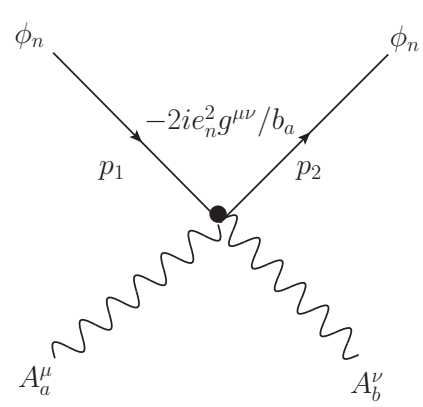

(b)

Fig 2 .

The propagator of the $a$-th vector field $A_{a, \mu}$ is

$$
\frac{b_{a}\left(g_{\mu \nu}+\frac{k_{\mu} k_{\nu}}{M_{a}^{2}}\right)}{k^{2}+M_{a}^{2}} \text {. }
$$

Due to the extra piece $k_{\mu} k_{\nu} / M_{a}^{2}$ in the propagator, the perturbation theory is non-renormalizable by naive power counting. But we will avoid the UV divergence by fine-tuned cancellation among all particles in the spectrum. Since all internal lines for vector fields are given by superpositions of all vector fields, the effect of introducing the massive vectors $A_{a, \mu}$ is equivalent to replacing the photon propagator by

$$
\Delta_{\mu \nu}(k)=\frac{b_{0}\left(g_{\mu \nu}-(1-\xi) \frac{k_{\mu} k_{\nu}}{k^{2}}\right)}{k^{2}}+\sum_{a=1}^{\infty} \frac{b_{a}\left(g_{\mu \nu}+\frac{k_{\mu} k_{\nu}}{M_{a}^{2}}\right)}{k^{2}+M_{a}^{2}}
$$

where $\xi$ is a free parameter depending on the choice of gauge. 


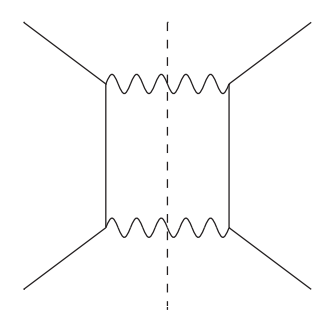

(a)

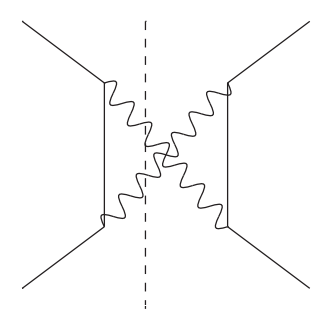

(b)

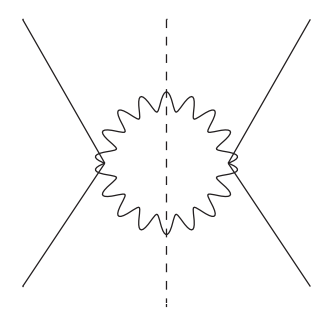

(c)

Fig 3 .

It should be noted that an equivalent form of Lorentz gauge appears as the divergence of the free field equation of $A_{a, \mu}$,

$$
\begin{aligned}
0 & =\partial_{\nu}\left[\frac{-1}{b_{a}}\left(\partial_{\mu} F_{a}^{\mu \nu}-M_{a}^{2} A_{a}^{\nu}\right)\right] \\
& =\frac{1}{b_{a}} M_{a}^{2} \partial_{\nu} A_{a}^{\nu} .
\end{aligned}
$$

The physical meaning to this condition is that a spin-one particle has only three physical polarizations.

The purpose of the rest of the section is to find the conditions on the parameters $c_{n}, e_{n}$, $m_{n}^{2}, b_{a}$ and $M_{a}^{2}$ such that this theory is UV-finite and unitary. We will first examine the conditions on unitarity, and then study in detail the UV divergence of several loop diagrams before concluding on generic diagrams.

\subsection{Unitarity}

In the previous subsection, we introduced massive vectors into our theory. Now we have to check that those massive vectors do not endanger unitarity.

First we briefly review how unitarity survives in massless vector field theories. The requirement that the S-matrix is unitary implies that the scattering amplitude $T_{i f}$ from the initial state $i$ to the final state $f$, defined as

$$
S_{i f}=\delta_{i f}+i(2 \pi)^{4} \delta\left(p_{i}-p_{f}\right) T_{i f}
$$

satisfies the relation

$$
T_{i f}-T_{f i}^{*}=i \sum_{n} T_{i n} T_{f n}^{*}(2 \pi)^{4} \delta\left(p_{i}-p_{f}\right)
$$

where the sum is taken over all physical states consistent with all conservation laws. The left hand side can be calculated purturbatively by means of the so-called Landau-Cutkosky rules [3]:

1. Cut through the diagram in all possible ways such that the cut propagators can simultaneously be put on shell. 


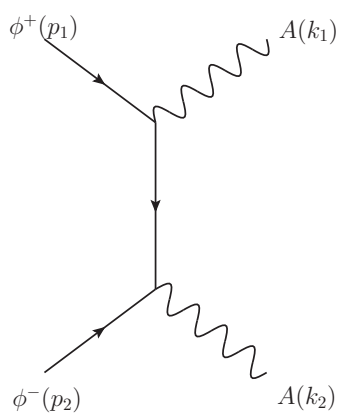

(a)

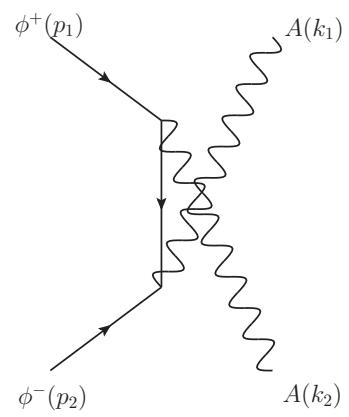

(b)

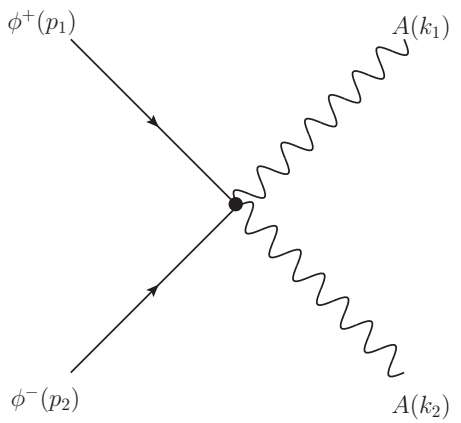

(c)

Fig 4 .

2. Replace $1 /\left(p^{2}+m^{2}+i \epsilon\right)$ by $-2 \pi i \delta\left(p^{2}+m^{2}\right)$ for each cut propagator, then perform the loop integrals.

3. Sum over the contributions of all possible cuts.

Using these rules, it is possible to check unitarity order by order in a perturbation theory. For example, Fig. 3 shows three possible ways of the interaction $\phi \phi \rightarrow \phi \phi$ of order $O\left(e^{4}\right)$. The Landau-Cutkosky rules include the replacement of the photon propagator in each internal line

$$
\Delta_{\mu \nu}=\frac{g_{\mu \nu}-(1-\xi) \frac{k_{\mu} k_{\nu}}{k^{2}}}{k^{2}+i \epsilon} \rightarrow-2 \pi i\left[g_{\mu \nu}-(1-\xi) \frac{k_{\mu} k_{\nu}}{k^{2}}\right] \delta\left(k^{2}\right) \equiv-2 \pi i G_{\mu \nu} \delta\left(k^{2}\right) .
$$

Therefore the left-hand side of (30) is

$$
\operatorname{Im} T=M^{\mu \rho} G_{\mu \nu} G_{\rho \xi} M^{\nu \xi}
$$

where $M^{\mu \rho}$ is an on-shell amplitude of $\phi \phi \rightarrow A A$ which contains three diagrams (Fig. 4.).

On the right-hand side of (30), the two diagrams obtained from cutting a diagram in Fig. (3) are multiplied. A cut vector internal line becomes two vector external lines in the two diagrams of the $\phi \phi \rightarrow A A$ process. The summation over polarizations of a pair of vector external lines contributes the factor

$$
\sum_{\lambda=1,2} \epsilon_{\mu}^{*}(\lambda, k) \epsilon_{\nu}(\lambda, k) \equiv-E_{\mu \nu}
$$

The sum only includes physical polarizations, so

$$
E_{\mu \nu}=g_{\mu \nu}-\frac{k_{\mu} n_{\nu}+k_{\nu} n_{\mu}}{n \cdot k}+\frac{n^{2} k_{\mu} k_{\nu}}{(k \cdot n)^{2}},
$$

where $n_{\nu}$ is an arbitrary vector satisfying

$$
k \cdot n \neq 0, \quad n \cdot \epsilon(\lambda, k)=0,
$$




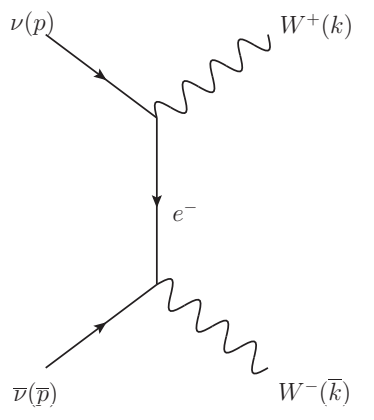

Fig 5 .

Compare (31) and (34). Their difference is that the unphysical polarizations are included in $G_{\mu \nu}$ but not in $E_{\mu \nu}$. However, in ordinary QED there is no problem due to the fact that photons always couple to conserved currents

$$
k_{\mu} M^{\mu \nu}=0
$$

Therefore,

$$
M^{\mu \rho} G_{\mu \nu} G_{\rho \xi} M^{\nu \xi}=M^{\mu \rho} E_{\mu \nu} E_{\rho \xi} M^{\nu \xi}=M^{\mu \rho} g_{\mu \nu} g_{\rho \xi} M^{\nu \xi}
$$

and unitarity is preserved.

In massive vector theories, the Ward-identity (36) no longer applies. Yet the situation is actually simpler. The sum over physical polarizations is

$$
\sum_{\lambda=1,2,3} \epsilon_{\mu}(\lambda, k) \epsilon_{\nu}^{*}(\lambda, k)=g_{\mu \nu}+\frac{k_{\mu} k_{\nu}}{M^{2}},
$$

which is exactly the numerator of the massive vector boson propagator. It means that there is no unphysical polarizations in the internal lines. In massive theories, the number of physical polarizations is three, and it is exactly the number of degrees of freedom of spin-one particles. On the other hand, for a massless vector field, in order to obtain a well-defined propagator, one introduces a gauge-fixing term of the form

$$
\frac{-1}{2 \xi}\left(\partial_{\mu} A^{\mu}\right)^{2}
$$

In the propagator the number of polarizations is 4 , but there are only 2 physical polarizations. Therefore, we need Ward-identities to ensure that unphysical intermediate states decouple.

A well known theory containing massive vector bosons is the intermediate vector boson (IVB) model of weak interaction. In this theory heavy vector bosons $W^{ \pm}$are introduced to tame the high energy behavior of beta decay in Fermi's four-fermion current-current model. But diagrams with external $W^{ \pm}$bosons $[5,6]$, e.g. Fig. 5, still suffer high energy divergence. 
At the leading order, the cross section is $[5,6]$

$$
\frac{d \sigma}{d \Omega}=\frac{\sum_{p o l}\left|M_{t}\right|^{2}}{64 \pi^{2} s} \simeq \frac{G^{2} E^{2} \sin ^{2} \theta}{8 \pi^{2}} .
$$

When the energy is large enough, the total probability of the scattering is larger than 1 , and unitarity is violated, in a sense different from our discussions above.

The murderer of unitarity here is the longitudinal mode of $W^{ \pm}$bosons. Recall the polarization sum (38). The second term in (38), which results in bad high-energy behaviors, comes from the longitudinal mode.

To cancel this $E^{2}$ term, destructive interference in s-channel (Fig 6(a)) and/or u-channel (Fig 6(b)) is a possible way out. This motivated the introduction of the $\mathrm{Z}$ boson.

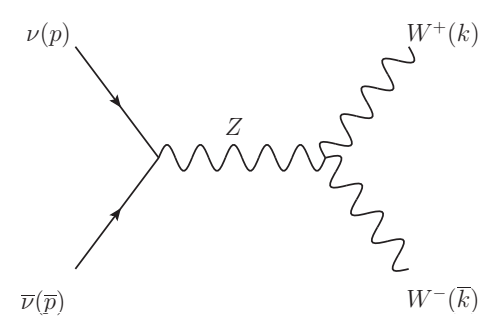

(a)

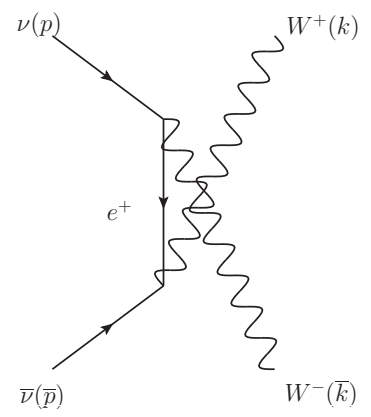

(b)

Fig 6 .

In our theory, we will also rely on the destructive interference among diagrams with the same external legs. For example, the pair annihilation $\phi_{n}^{+} \phi_{n}^{-} \rightarrow A_{a} A_{b}$ contains three diagrams at the tree level (Fig. 7), the amplitude is

$$
\mathcal{T}=-\frac{e_{n}^{2}}{c_{n}}\left[\frac{4\left(p_{1} \cdot \epsilon_{1}\right)\left(p_{2} \cdot \epsilon_{2}\right)}{m_{n}^{2}-t}+\frac{4\left(p_{1} \cdot \epsilon_{2}\right)\left(p_{2} \cdot \epsilon_{1}\right)}{m_{n}^{2}-u}+2\left(\epsilon_{1} \cdot \epsilon_{2}\right)\right],
$$

where

$$
t=-\left(-p_{1}-k_{1}\right)^{2}, \quad \text { and } \quad u=-\left(-p_{1}-k_{2}\right)^{2} .
$$

Here the incoming momenta are defined to have negative energy and the relation of energy momentum conservation is

$$
p_{1}+p_{2}+k_{1}+k_{2}=0 .
$$

Because we only worry about the $E^{4}$ term in $|\mathcal{T}|^{2}$, we focus our attention on leading order terms in the expansion

$$
\begin{aligned}
& \frac{1}{m_{n}^{2}-t}=\frac{1}{m_{n}^{2}+\left(p_{1}+k_{1}\right)^{2}}=\frac{1}{2\left(p_{1} \cdot k_{1}\right)-M_{a}^{2}}=\frac{1}{2 p_{1} \cdot k_{1}}\left[1+\mathcal{O}\left(1 / E^{2}\right)\right] \\
& \frac{1}{m_{n}^{2}-u}=\frac{1}{m_{n}^{2}+\left(p_{1}+k_{2}\right)^{2}}=\frac{1}{2\left(p_{1} \cdot k_{2}\right)-M_{b}^{2}}=\frac{1}{2 p_{1} \cdot k_{2}}\left[1+\mathcal{O}\left(1 / E^{2}\right)\right]
\end{aligned}
$$




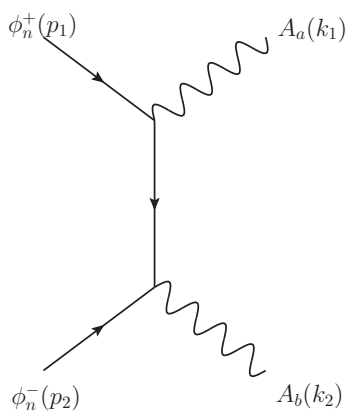

(a)

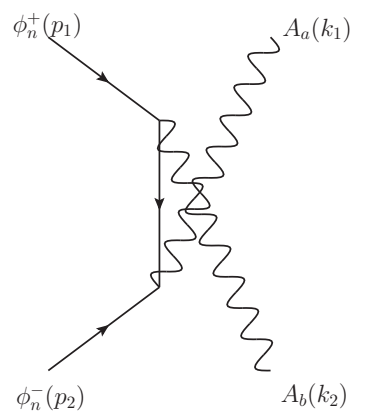

(b)

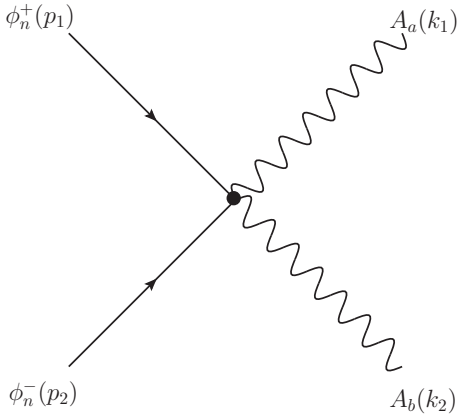

(c)

Fig 7.

and the longitudinal polarizations are

$$
\epsilon_{1}^{\mu} \rightarrow \frac{k_{1}^{\mu}}{M_{a}}, \quad \epsilon_{2}^{\mu} \rightarrow \frac{k_{2}^{\mu}}{M_{b}} .
$$

Using these relations, the high energy behavior of the amplitude in (41) is largely simplified

$$
\mathcal{T} \rightarrow-\frac{e_{n}^{2}}{c_{n}}\left[\frac{2\left(p_{2} \cdot k_{2}\right)}{M_{a} M_{b}}+\frac{2\left(p_{2} \cdot k_{1}\right)}{M_{a} M_{b}}+2 \frac{\left(k_{1} \cdot k_{2}\right)}{M_{a} M_{b}}\right]=\frac{-e_{n}^{2}}{c_{n}} \frac{M_{a}^{2}+M_{b}^{2}}{M_{a} M_{b}} .
$$

The term of order $E^{2}$ is suppressed due to the identity

$$
s+t+u=2 m_{n}^{2}+M_{a}^{2}+M_{b}^{2} .
$$

We see in (46) the destructive interference among three diagrams. Unitarity is safe at high energies.

Next we consider the $\phi_{m}^{+} \phi_{m}^{-} \rightarrow \phi_{n}^{+} \phi_{n}^{-}$diagram (Fig. 8). The amplitude is

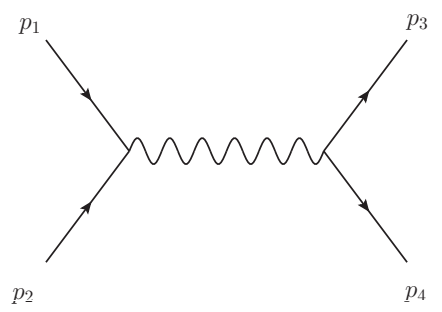

Fig 8 .

$$
\mathcal{M}_{m m n n}=-i e_{m}\left(p_{1}-p_{2}\right)^{\mu} \sum_{a} \frac{b_{a}\left(g_{\mu \nu}+\frac{\left(p_{1}+p_{2}\right)_{\mu}\left(p_{3}+p_{4}\right)_{\nu}}{M_{a}^{2}}\right)}{M_{a}^{2}-s}\left(-i e_{n}\right)\left(p_{3}-p_{4}\right)^{\nu} .
$$

Although the term $k^{\mu} k^{\nu} / M^{2}$ in the propagator leads to a term of 4 th order in energy, it vanishes because

$$
\left(p_{1}-p_{2}\right)^{\mu}\left(p_{1}+p_{2}\right)_{\mu}\left(p_{3}+p_{4}\right)_{\nu}\left(p_{3}-p_{4}\right)^{\nu}=\left(p_{1}^{2}-p_{2}^{2}\right)\left(p_{3}^{2}-p_{4}^{2}\right)=0 .
$$


Instead of imposing the equation of motion on (48), there is another way to control the $k^{\mu} k^{\nu}$ term in propagator. Take the $1 / E$ expansion of (48),

$$
\mathcal{M}_{m m n n}=-i e_{m}\left(p_{1}-p_{2}\right)^{\mu} \sum_{a}\left[-b_{a} \frac{\left(p_{1}+p_{2}\right)_{\mu}\left(p_{3}+p_{4}\right)_{\nu}}{M_{a}^{2} s}+\mathcal{O}\left(1 / E^{2}\right)\right]\left(-i e_{n}\right)\left(p_{3}-p_{4}\right)^{\nu}
$$

the leading order term vanishes if the condition

$$
\sum_{a=1} \frac{b_{a}}{M_{a}^{2}}=0
$$

is imposed. Although this is not necessary here, we will meet the same condition in the next subsection in order for certain loop diagrams to be finite.

In general, the potential violation of unitarity at high energies due to massive vector bosons has its origin in the $k_{\mu} k_{\nu} / M_{a}^{2}$ term in the propagator. The same term is also responsible for potential nonrenormalizability. Our basic idea is to fine-tune the parameters $b_{a}, M_{a}^{2}$ to achieve destructive interference among all massive vector bosons, such that the vector propagator is sufficiently well-behaved at high energies for the theory to be UV-finite and unitary.

\subsection{UV divergences in loop diagrams}

In this subsection we calculate some one-loop diagrams, and show that UV divergences are canceled in every diagram if certain conditions on the parameters of the theory are matched. Then we discuss the cancellation in generic Feynman diagrams.

\subsubsection{Self-energy of $\phi$}

We first calculate the self-energy diagram for the scalars. The two external lines must have the same index $n$. There are two diagrams contributing to self-energy as shown in Fig. 9.

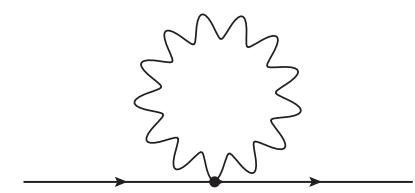

(a)

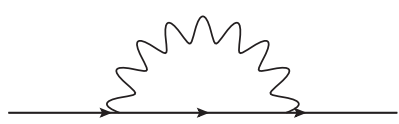

(b)

Fig 9 .

The amplitude for Fig. 9(a) is

$$
\mathcal{M}_{n n}^{(1)}=\left(-2 i \frac{e_{n}^{2}}{c_{n}} g^{\mu \nu}\right)\left(\frac{1}{i}\right) \int \frac{d^{4} l}{\left(2 \pi^{4}\right)}\left[\frac{b_{0}\left(g_{\mu \nu}-(1-\xi) \frac{l_{\mu} l_{\nu}}{l^{2}}\right)}{l^{2}}+\sum_{a=1}^{\infty} \frac{b_{a}\left(g_{\mu \nu}+\frac{l_{\mu} l_{\nu}}{M_{a}^{2}}\right)}{l^{2}+M_{a}^{2}}\right] .
$$


The term with the worst UV-divergence is

$$
\mathcal{M}_{n n}^{\prime} \propto \sum_{a=1}^{\infty} \frac{1}{M_{a}^{2}} \int d^{4} l \frac{b_{a} l^{2}}{l^{2}+M_{a}^{2}} \propto \Lambda^{4}
$$

for UV cutoff at $\Lambda$. In general we also expect UV divergences of lower orders. It is easy to see that, in the gauge $\xi=0,{ }^{3} \mathrm{UV}$ divergences at various orders are proportional to

$$
\sum_{a=1}^{\infty} \frac{1}{M_{a}^{2}} b_{a} \Lambda^{4}, \quad\left(b_{0}+\sum_{a=1}^{\infty} \frac{1}{M_{a}^{2}} b_{a} M_{a}^{2}\right) \Lambda^{2}, \quad \sum_{a=1}^{\infty} \frac{1}{M_{a}^{2}} b_{a} M_{a}^{4} \log \left(\Lambda^{2}\right) .
$$

Therefore, at least three conditions are required to remove all UV divergences:

$$
\begin{aligned}
\sum_{a=1}^{\infty} \frac{b_{a}}{M_{a}^{2}} & =0 \\
\sum_{a=0}^{\infty} b_{a} & =0 \\
\sum_{a=1}^{\infty} b_{a} M_{a}^{2} & =0 .
\end{aligned}
$$

By imposing the condition (57a), we find something interesting in the vector propagator. Calculations below will also be done in the gauge $\xi=0$, which is more convenient. In this gauge, the propagator (27) becomes

$$
\begin{aligned}
\Delta_{\mu \nu}(k) & =\frac{b_{0}\left(g_{\mu \nu}-\frac{k_{\mu} k_{\nu}}{k^{2}}\right)}{k^{2}}+\sum_{a=1}^{\infty} \frac{b_{a}\left(g_{\mu \nu}+\frac{k_{\mu} k_{\nu}}{M_{n}^{2}}\right)}{k^{2}+M_{n}^{2}} \\
& =\sum_{a=0}^{\infty} \frac{b_{a}\left(g_{\mu \nu}-\frac{k_{\mu} k_{\nu}}{k^{2}}\right)}{k^{2}+M_{n}^{2}}+\sum_{a=1}^{\infty} \frac{b_{a} k_{\mu} k_{\nu}}{k^{2}+M_{n}^{2}}\left(\frac{1}{M_{n}^{2}}+\frac{1}{k^{2}}\right) \\
& =\sum_{a=0}^{\infty} \frac{b_{a} P_{\mu \nu}}{k^{2}+M_{n}^{2}}+\frac{k_{\mu} k_{\nu}}{k^{2}}\left(\sum_{a=1} \frac{b_{a}}{M_{n}^{2}}\right) \\
& =\sum_{a=0}^{\infty} \frac{b_{a} P_{\mu \nu}}{k^{2}+M_{n}^{2}} .
\end{aligned}
$$

\footnotetext{
${ }^{3}$ For our purpose, the gauge $\xi=0$ is particularly convenient. In generic gauge, the UV divergence of order $\Lambda^{2}$ for the diagram in Fig. 9(a) is actually proportional to

$$
(1-(1-\xi) / 4) b_{0}+(1-1 / 4) \sum_{a=1}^{\infty} b_{a}=0 .
$$

On the other hand, the diagram in Fig. 9(b) contributes a factor of

$$
-\frac{1}{4}(1-(1-\xi)) b_{0}
$$

to the same divergent term. The sum of the two diagrams is gauge invariant.
} 
Here

$$
P_{\mu \nu} \equiv g_{\mu \nu}-\frac{k_{\mu} k_{\nu}}{k^{2}}
$$

is a projection that projects out vectors parallel to $k$. Therefore, by imposing the condition (57a), the polarization of a vector propagator is perpendicular to its momentum even off-shell!

Now we can rewrite (52) in the form

$$
\mathcal{M}_{n n}^{(1)}=\left(-2 i \frac{e_{i}^{2}}{c_{n}} g^{\mu \nu}\right)\left(\frac{1}{i}\right) \sum_{a=0} \int \frac{d^{d} l}{(2 \pi)^{d}} \frac{b_{a} P_{\mu \nu}}{k^{2}+M_{a}^{2}} .
$$

Integrating it in $d=4-\epsilon$ dimensions gives

$$
\begin{aligned}
\mathcal{M}_{n n}^{(1)} & =-2 \frac{e_{n}^{2}}{c_{n}}(d-1) \sum_{a=0} \int \frac{d^{d} l}{(2 \pi)^{d}} \frac{b_{a}}{k^{2}+M_{a}^{2}} \\
& =-2 \frac{e_{n}^{2}}{c_{n}}(3-\epsilon) \sum_{a=0} \frac{b_{a} M_{a}^{2}}{(4 \pi)^{2-\epsilon / 2}}\left[\frac{-2}{\epsilon}+\gamma-1+\mathcal{O}(\epsilon)\right]\left[1+\frac{\epsilon}{2} \log \left(\frac{1}{M_{a}^{2}}\right)+\mathcal{O}\left(\epsilon^{2}\right)\right] .
\end{aligned}
$$

The coefficient of the $1 / \epsilon$ term vanishes if the condition $(57 \mathrm{c})$ is satisfied. The finite part of the scattering amplitude can be obtained by taking the limit $\epsilon \rightarrow 0$,

$$
\mathcal{M}_{n n}^{(1)}=-\frac{3 e_{n}^{2}}{8 \pi^{2} c_{n}^{2}} \sum_{a=0} b_{a} M_{a}^{2} \log \left(M_{a}^{2}\right) .
$$

In the sum over the index $a$, the term for $a=0$ is ill-defined. This is the same infrared divergence in ordinary QED. It is resulted from the ignorance of diagrams with soft photons $(k=0)$. A simple way to deal with it is to introduce a mass term $m_{\gamma}$ to the photon as an infrared regulator and take the limit $m_{\gamma} \rightarrow 0$ at the end. The final result is that the $a=0$ term has no contribution.

Now we calculate the amplitude for Fig. 9(b). It is

$$
\begin{aligned}
\mathcal{M}_{n n}^{(2)} & =\left(\frac{i e_{n}}{c_{n}}\right)^{2}\left(\frac{1}{i}\right)^{2} \int \frac{d^{4} l}{(2 \pi)^{4}}(l+2 p)^{\mu} \frac{c_{n}}{(l+p)^{2}+m_{n}^{2}}(l+2 p)^{\nu} \sum_{a=0}\left(\frac{b_{a} P_{\mu \nu}(l)}{l^{2}+M_{a}^{2}}\right) \\
& =e_{n}^{2}(2 p)^{\mu}(2 p)^{\nu} \frac{1}{c_{n}} \sum_{a=0} b_{a} \int \frac{d^{d} l}{(2 \pi)^{d}} \frac{1}{(l+p)^{2}+m_{n}^{2}} \frac{P_{\mu \nu}(l)}{l^{2}+M_{a}^{2}} .
\end{aligned}
$$

Multiplying the numerator and denominator in (63) by $l^{2}$ and using Feynman's parameters, we can compute the essential part of the integral above as

$$
\int \frac{d^{d} l}{(2 \pi)^{d}} \frac{l^{2} P_{\mu \nu}(l) p^{\mu} p^{\nu}}{l^{2}\left(l^{2}+M_{a}^{2}\right)\left((l+p)^{2}+m_{n}^{2}\right)}=\int d F_{3} \int \frac{d^{d} l}{(2 \pi)^{d}} \frac{N}{\left(q^{2}+\Delta\right)^{3}},
$$


where

$$
\begin{aligned}
\int d F_{3} & \equiv 2 \int_{0}^{1} \int_{0}^{1} \int_{0}^{1} d \alpha_{1} d \alpha_{2} d \alpha_{3} \delta\left(\alpha_{1}+\alpha_{2}+\alpha_{3}-1\right), \\
q & =\alpha_{1} l+\alpha_{2} l+\alpha_{3}(l+p)=l+\alpha_{3} p, \\
\Delta & =\alpha_{3}\left(1-\alpha_{3}\right) p^{2}+\alpha_{2} M_{a}^{2}+\alpha_{3} m_{n}^{2}, \\
N & =q^{2} p^{2}-d^{-1} q^{2} p^{2} .
\end{aligned}
$$

Again we apply dimensional regularization to define the integral

$$
\sum_{a=0} b_{a} \int \frac{d^{d} l}{(2 \pi)^{d}} \frac{q^{2}}{\left(q^{2}+\Delta\right)^{3}} \approx \sum_{a=0} b_{a}\left[\frac{2}{\epsilon}-\log \Delta-\gamma+\mathcal{O}(\epsilon)\right] \frac{1}{(4 \pi)^{2}} .
$$

The $1 / \epsilon$ term vanishes with the condition (57b). The finite part is

$$
\mathcal{M}_{n n}^{(2)}=-\frac{6 e_{n}^{2}}{c_{n}} \int_{0}^{1} \prod_{i=1}^{3} d \alpha_{i} \delta\left(\alpha_{1}+\alpha_{2}+\alpha_{3}-1\right) \sum_{a=0} b_{a} \log \left[\alpha_{3}\left(1-\alpha_{3}\right) p^{2}+\alpha_{2} M_{a}^{2}+\alpha_{3} m_{n}^{2}\right] .
$$

\subsubsection{Choice of parameters}

We can construct a solution of parameters to all the conditions (57) in a way similar to (8). Because $M_{0}^{2}=0$, for convenience we rewrite (57) in the form

$$
\begin{aligned}
& \sum_{a=1}^{\infty} \frac{b_{a}}{M_{a}^{2}}=0, \\
& \sum_{a=1}^{\infty} b_{a}=-b_{0}, \\
& \sum_{a=1}^{\infty} b_{a} M_{a}^{2}=0 .
\end{aligned}
$$

A special class of solutions of the parameters is

$$
\begin{aligned}
b_{a} & =\left[a+1+x_{1}(a+2)(a+1)+x_{2}(a+1)(a+2)(a+3)\right] e^{z a} \quad\left(a \geq 1, x_{i} \geq 0\right), \\
M_{a}^{2} & =e^{\alpha a} \quad(a \geq 1, \alpha>0) .
\end{aligned}
$$

The value of $b_{0}$ will be determined by $(71 \mathrm{~b})$, and $M_{0}$ is 0 . In addition to the reality condition $M_{a}^{2}>0$, we also need $b_{a}>0$ for all $a=0,1,2, \cdots$ for the sake of unitarity. The values of $z$ and $\alpha$ will be set to be positive numbers.

That the solution above satisfies all conditions can be seen as follows. Let $\rho \equiv e^{z+\alpha r}, r=$ \pm 1 . We compute $\sum b_{a} M_{a}^{2 r}$ assuming $\rho<1$ and then analytically continue $\rho$ back to $\rho>1$. We get

$$
\begin{aligned}
\sum_{a=1}^{\infty} b_{a} M_{a}^{2 r} & =\frac{d}{d \rho}\left(\frac{1}{1-\rho}\right)+x_{1} \frac{d^{2}}{d \rho^{2}}\left(\frac{1}{1-\rho}\right)+x_{2} \frac{d^{3}}{d \rho^{3}}\left(\frac{1}{1-\rho}\right) \\
& =\frac{1}{\xi^{2}}+\frac{x_{1}}{\xi^{3}}+\frac{x_{2}}{\xi^{4}} \equiv h(\xi),
\end{aligned}
$$


where $\xi \equiv \frac{1}{1-\rho}$, which is negative definite when $\rho>1$. By this method, we have sufficient parameters $\left\{x_{1}, x_{2}\right\}$ to fix the root of $\xi$ at desired positions. Defining the roots as

$$
-\left|\xi_{1}\right|=\frac{1}{1-e^{z-\alpha}}, \quad-\left|\xi_{2}\right|=\frac{1}{1-e^{z}}, \quad-\left|\xi_{3}\right|=\frac{1}{1-e^{z+\alpha}} .
$$

We see that $\left|\xi_{3}\right|>\left|\xi_{2}\right|>\left|\xi_{1}\right|>0$. The corresponding values of $x_{1}$ and $x_{2}$ can be found by simply comparing the coefficients with the following equation

$$
\xi^{4} h(\xi)=c\left(\xi+\left|\xi_{1}\right|\right)\left(\xi+\left|\xi_{3}\right|\right)
$$

where $c$ is an arbitrary positive real parameter. Both $x_{1}$ and $x_{2}$ are guaranteed to be positive because no negative coefficient appears in (75). Furthermore, $h\left(-\left|\xi_{2}\right|\right)$ is negative so that we let

$$
b_{0}=-h\left(-\left|\xi_{2}\right|\right)>0 .
$$

The finite part of a diagram is free from UV divergence, but it is not necessarily finite because it involves an infinite series. Ignoring the integration of Feynman parameters, $\mathcal{M}_{n n}^{(1)}$ and $\mathcal{M}_{n n}^{(2)}$ are both of the form

$$
\sum_{a_{1} \cdots a_{I}} b_{a_{1}} \cdots b_{a_{I}} \Delta^{r} \log \Delta
$$

The magnitude of $\Delta$ is roughly of the same order as $M_{a}^{2}$, which grows exponentially with $a$, so $\log \Delta$ grows like $a$ when $a$ increases. On the other hand, $b_{a}$ decays exponentially with $a$ for $z<0$. For $z$ sufficiently negative, the series converges. The next step is to analytically continue $z$ to $z_{0}>0$. This might lead to new divergence. But it is free from divergences as long as

$$
m z_{0}-n \alpha \neq 0 \quad \forall m, n \in \mathbb{Z} .
$$

That is, we want the ratio $z_{0} / \alpha$ to be an irrational number. This condition can be understood as the following: this choice of parameters (71) is based on the analytic continuation of geometric series. But the series can not be defined even by analytic continuation when the ratio between successive terms is equal to one. To prevent this for a generic series of the form (77), the condition

$$
e^{m z_{0}-n a} \neq 1 \quad \forall m, n \in \mathbb{Z}
$$

is sufficient.

\subsubsection{Self-energy of $A_{a, \mu}$}

Now we calculate the vector field's self-energy. The one-loop corrections to the vector propagator are shown in Fig 10. 


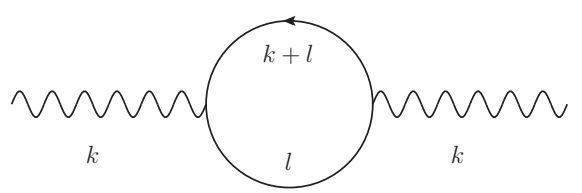

(a)

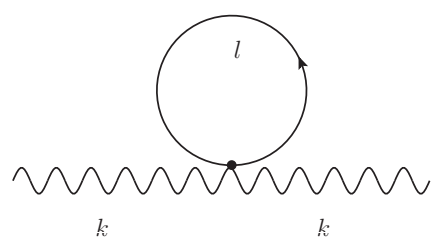

(b)

Fig 10.

The sum of these two diagrams is

$$
\begin{aligned}
\Pi^{\mu \nu}(k) & =\left(\frac{1}{i}\right)^{2} \sum_{n}\left(\frac{i e_{n}}{c_{n}}\right)^{2} \int \frac{d^{4} l}{(2 \pi)^{4}}(2 l+k)^{\mu} \frac{c_{n}}{l^{2}+m_{n}^{2}}(2 l+k)^{\nu} \frac{c_{n}}{(l+k)^{2}+m_{n}^{2}} \\
& +(-2 i) g^{\mu \nu}\left(\frac{1}{i}\right) \sum_{n} \frac{e_{n}^{2}}{c_{n}} \int \frac{d^{4} l}{(2 \pi)^{4}} \frac{c_{n}}{l^{2}+m_{n}^{2}} \\
& =\sum_{n} e_{n}^{2} \int \frac{d^{4} l}{(2 \pi)^{4}}(2 l+k)^{\mu} \frac{1}{l^{2}+m_{n}^{2}}(2 l+k)^{\nu} \frac{1}{(l+k)^{2}+m_{n}^{2}} \\
& +(-2) g^{\mu \nu} \sum_{n} e_{n}^{2} \int \frac{d^{4} l}{(2 \pi)^{4}} \frac{1}{l^{2}+m_{n}^{2}} .
\end{aligned}
$$

As all factors of $c_{n}$ 's cancel, the UV divergence can only be cancelled by tuning the coupling constants $e_{n}$.

The first term in (80) can be written in the form

$$
\begin{aligned}
\Pi^{(1) \mu \nu}(k) & =\sum_{n} e_{n}^{2} \int \frac{d^{4} l}{(2 \pi)^{4}} \frac{(2 l+k)^{\mu}(2 l+k)^{\nu}}{\left[(l+k)^{2}+m_{n}^{2}\right]\left[l^{2}+m_{n}^{2}\right]} \\
& =\int_{0}^{1} d \alpha \sum_{n} e_{n}^{2} \int \frac{d^{4} q}{(2 \pi)^{4}} \frac{N^{\mu \nu}}{\left(q^{2}+\Delta_{n n}\right)^{2}}
\end{aligned}
$$

where

$$
\begin{aligned}
q & =\alpha(l+k)+(1-\alpha) l=l+\alpha k, \\
\Delta_{n n} & =m_{n}^{2}+\alpha(1-\alpha) k^{2}, \\
N^{\mu \nu} & =g^{\mu \nu} q^{2}+(1-2 \alpha)^{2} k^{\mu} k^{\nu} .
\end{aligned}
$$

In $d=4-\epsilon$ dimensions, (81) can be calculated

$$
\begin{aligned}
\Pi^{(1) \mu \nu}(k)= & \int_{0}^{1} d \alpha \sum_{n} 2 e_{n}^{2} \frac{g^{\mu \nu} \Delta_{n n}}{16 \pi^{2}}\left[\frac{-2}{\epsilon}+\gamma-1+\mathcal{O}(\epsilon)\right]\left[1+\frac{\epsilon}{2} \log \left(\frac{1}{\Delta_{n n}}\right)+\mathcal{O}\left(\epsilon^{2}\right)\right] \\
& +\int_{0}^{1} d \alpha \sum_{n} e_{n}^{2} \frac{(1-2 \alpha)^{2} k^{\mu} k^{\nu}}{16 \pi^{2}}\left[\frac{2}{\epsilon}-\log \Delta_{n n}-\gamma+\mathcal{O}(\epsilon)\right] .
\end{aligned}
$$


For the $1 / \epsilon$ terms in (83) to vanish, we need two conditions

$$
\begin{aligned}
& \sum_{n=0}^{\infty} e_{n}^{2}=0, \\
& \sum_{n=0}^{\infty} e_{n}^{2} m_{n}^{2}=0 .
\end{aligned}
$$

These are reminiscent of those in (2), with $c_{n}$ 's replaced by $e_{n}$ 's.

The finite part of this amplitude is

$$
\Pi^{(1) \mu \nu}(k)=\frac{g^{\mu \nu}}{8 \pi^{2}} \int_{0}^{1} d \alpha \sum_{n=0}^{\infty} e_{n}^{2} \Delta_{n n} \log \Delta_{n n}-\int_{0}^{1} d \alpha \sum_{n=0}^{\infty} e_{n}^{2} \frac{(1-2 \alpha)^{2} k^{\mu} k^{\nu}}{16 \pi^{2}} \log \Delta_{n n} .
$$

The second term in $(80)$ is

$$
\Pi^{(2) \mu \nu}(k)=(-2) g^{\mu \nu} \sum_{n} e_{n}^{2} \int \frac{d^{4} l}{(2 \pi)^{4}} \frac{1}{l^{2}+m_{n}^{2}} .
$$

The calculation is straightforward. UV-divergences cancel due to (84), and the finite part is

$$
\Pi^{(2) \mu \nu}(k)=-\frac{g^{\mu \nu}}{8 \pi^{2}} \sum_{n=0}^{\infty} e_{n}^{2} m_{n}^{2} \log \left(m_{n}^{2}\right) .
$$

Integrating over the Feynman parameter in (85) and adding it to (87), we compute the self-energy of $A_{a, \mu}$ as

$$
\Pi^{\mu \nu}(k)=\frac{g^{\mu \nu} k^{2}-k^{\mu} k^{\nu}}{8 \pi^{2}} \sum_{n=0}^{\infty} e_{n}^{2}\left[\frac{\sqrt{\frac{\left(k^{2}+4 m_{n}^{2}\right)^{3}}{3 k^{2}}} \tanh ^{-1}\left(\sqrt{\frac{k^{2}}{k^{2}+4 m_{n}^{2}}}\right)}{k^{2}}+\frac{\log m_{n}}{3}\right] .
$$

In order for the $U(1)$ gauge symmetry to be valid at the quantum level, the photon must remain massless against quantum corrections. The mass correction of the photon can be derived from its self-energy. Eq. (88) only provides its finiteness. We need to check that the mass correction vanishes.

At one loop, the vector propagator is given by

$$
\Delta_{\nu}^{\mu}(k)=\Delta_{\nu}^{\mu}+\Delta_{\alpha_{1}}^{\mu} \Pi_{\alpha_{2}}^{\alpha_{1}} \Delta_{\nu}^{\alpha_{2}}+\Delta_{\alpha_{1}}^{\mu} \Pi_{\alpha_{2}}^{\alpha_{1}} \Delta_{\alpha_{3}}^{\alpha_{2}} \Pi_{\alpha_{4}}^{\alpha_{3}} \Delta_{\nu}^{\alpha_{4}}+\cdots
$$

Let us define a scalar function $\Delta_{0}(k)$ in the photon propagator for convenience

$$
\Delta_{0}^{\mu \nu}(k)=P^{\mu \nu} \Delta_{0}(k),
$$

where

$$
\Delta_{0}(k) \equiv \frac{b_{0}}{k^{2}} .
$$




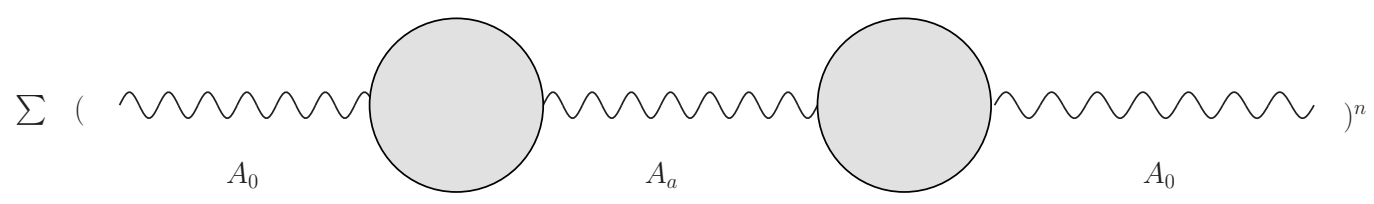

Fig 11.

Similarly, we rewrite (88) as

$$
\Pi^{\mu \nu}(k)=\frac{P^{\mu \nu}}{8 \pi^{2}} k^{2} \Pi(k)
$$

where

$$
\Pi(k) \equiv \sum_{n=0}^{\infty} \frac{e_{n}^{2}}{8 \pi^{2}}\left[\frac{\sqrt{\frac{\left(k^{2}+4 m_{n}^{2}\right)^{3}}{k^{2}}} \tanh ^{-1}\left(\sqrt{\frac{k^{2}}{k^{2}+4 m_{n}^{2}}}\right)}{3 k^{2}}+\frac{\log m_{n}}{3}\right] .
$$

Note that only the massless propagator $\Delta_{0}^{\mu \nu}(k)$ appears in internal lines. The diagram in Fig. 11 with $n \neq 0$ is considered a two-loop correction. The infinite series in (89) can be largely simplified as

$$
\begin{aligned}
\boldsymbol{\Delta}_{0, \nu}^{\mu}(k) & =P_{\nu}^{\mu}\left(\Delta_{0}(k)+\Delta_{0} k^{2} \Pi(k) \Delta_{0}(k)+\Delta_{0} k^{2} \Pi(k) \Delta_{0}(k) k^{2} \Pi(k) \Delta_{0}(k)+\cdots\right) \\
& =P_{\nu}^{\mu} \frac{b_{0}}{k^{2}-b_{0} k^{2} \Pi(k)} .
\end{aligned}
$$

In the neighborhood of the pole $k^{2}=0$, the propagator is approximated by

$$
\Delta_{0, \nu}^{\mu}(k) \simeq P_{\nu}^{\mu}(0) \frac{b_{0}}{k^{2}-b_{0}\left[\lim _{k^{2} \rightarrow 0} k^{2} \Pi(k)\right]},
$$

and so the mass correction is

$$
\delta m_{\gamma}^{2}=\lim _{k^{2} \rightarrow 0} b_{0} k^{2} \Pi(k),
$$

Using (93) and the condition (84b), we compute the mass correction as

$$
\begin{aligned}
\delta m_{\gamma}^{2} & =\lim _{k^{2} \rightarrow 0} \sum_{n=0} \frac{e_{n}^{2}}{24 \pi^{2}}\left[\sqrt{\frac{\left(k^{2}+4 m_{n}^{2}\right)^{3}}{k^{2}}} \tanh ^{-1}\left(\sqrt{\frac{k^{2}}{k^{2}+4 m_{n}^{2}}}\right)+k^{2} \log m_{n}\right] \\
& =\sum_{n=0} \frac{e_{n}^{2}}{24 \pi^{2}} \times 4 m_{n}^{2} \\
& =0 .
\end{aligned}
$$

We find that the mass correction of photon is indeed zero. 


\subsection{Generic Feynman diagrams}

Here we discuss the finiteness of generic Feynman diagrams. We will show that the power of superficial divergence $D$ of all Feynman diagrams are negative.

Define $L$ as the number of loops, $I_{A} / E_{A}$ as the number of vector internal/external lines, $I_{\phi} / E_{\phi}$ as the number of scalar internal/external lines, $V_{3}$ as the number of the vertices $\phi \phi A$, $V_{4}$ as the number of the vertices $\phi \phi A A$. These parameters satisfy several algebraic relations for all diagrams. First, we have

$$
L=I_{\phi}+I_{A}-V_{3}-V_{4}+1
$$

This equality states that the number of free momenta (on the loops) is equal to the number of internal lines minus the constraints due to energy-momentum conservation. For each vertex, there is a constraint on energy-momentum conservation, but that for the external momenta is irrelevant for the loop momenta.

Each vertex $\phi \phi A$ has only one leg of vector, and each vertex $\phi \phi A A$ has two. Therefore,

$$
2 V_{4}+V_{3}=2 I_{A}+E_{A} .
$$

Similarly, both vertices $\phi \phi A$ and $\phi \phi A A$ have two legs of scalar,

$$
2 V_{4}+2 V_{3}=2 I_{\phi}+E_{\phi}
$$

In the previous subsections, we saw that vector internal lines can always be treated as a superposition of all vector particles, while the scalar internal lines cannot. The effective vector propagator is $(58)$

$$
\begin{aligned}
\Delta_{\mu \nu} & =\sum_{a=0}^{\infty} \frac{b_{a} P_{\mu \nu}}{k^{2}+M_{a}^{2}} \\
& =P_{\mu \nu}\left(\frac{\sum_{a} b_{a}}{k^{2}}-\frac{\sum_{a} b_{a} M_{a}^{2}}{k^{4}}+\frac{\sum_{a} b_{a} M_{a}^{4}}{k^{6}}+\cdots\right) \\
& =\sum_{a=0}^{\infty} \frac{b_{a} P_{\mu \nu} M_{a}^{4}}{k^{4}\left(k^{2}+M_{a}^{2}\right)}
\end{aligned}
$$

which goes like $1 / k^{6}$ at high energies. The power of superficial UV divergence of an arbitrary diagram is

$$
D=4 L+V_{3}-2 I_{\phi}-6 I_{A}
$$

Each loop integral contributes a 4 dimensional integral of energy-momentum, the propagator of $A$ a factor of $1 / k^{6}$ and the propagator of $\phi$ a factor of $1 / k^{2}$ at high energies. Furthermore each vertex $\phi \phi A$ contributes a linear power of momentum due to the coupling.

Combining (98), (99), (100) and (102), we get

$$
D=4-4 I_{A}-E_{A}-E_{\phi}
$$


The superficial divergence is bounded from above, as in $\phi^{4}$ theory. With the assumption that the superficial divergence reflects the real power of divergence, UV finiteness is guaranteed if

$$
I_{A}>1
$$

When $I_{A}=1, D$ is non-negative only if there is no external lines of $A$ nor $\phi$. The only such possibility is then a vacuum diagram composed of a single loop of $A$. This diagram actually has $D=-2$ and is free from UV divergence. When $I_{A}=0$, that is, all vectors are external, all loops are composed only of scalar internal lines. A loop of scalar is divergent only when it is composed of no more than two scalar internal lines. These cases are already studied earlier when we computed the self-energy of the vector field (Fig. 10). They are also UV finite. Thus we believe that all diagrams of the theory are UV finite.

\section{Discussion}

\subsection{Self interactions of $\phi$}

In the above we assumed that the self interactions of the scalars vanish. That is,

$$
V\left(\sum_{n} \phi_{n}^{\dagger} \phi_{n}\right)=0
$$

We briefly discuss here the situation when $V \neq 0$.

Because of the global symmetry (25), a scalar propagator is not always superposed over all $\phi_{n}$, and so it has no suppression due to the conditions (2) at high energies. Even in 4 dimensions, the conditions (2) no longer ensure the finiteness of a loop consisting of only scalar interaction vertices.

Actually we have already seen the effect of the global symmetry (25) above. For example, in the diagram for self-energy of $\phi_{n}$ (Fig. 9), the index of the scalar internal line is fixed by the external lines. Similarly, in the self-energy diagram Fig. 10(a), the index $n$ of the two scalar propagators are not independent, and the UV divergence can not be cancelled by tuning the values of $c_{n}$. As we have seen earlier, these diagrams are UV-finite if the conditions (57) and (84) are satisfied. But what happens if the propagator of $A$ is replaced by a propagator of $\phi_{n}$, when the vertices are replaced by scalar self interactions?

It turns out that there are Feynman diagrams (e.g. Fig. 12) with logarithmic divergence which can not be removed by any condition on the coefficients because all the indices are fixed by the external lines. Therefore the assumption $V=0$ is a necessity for the sake of UV convergence. 


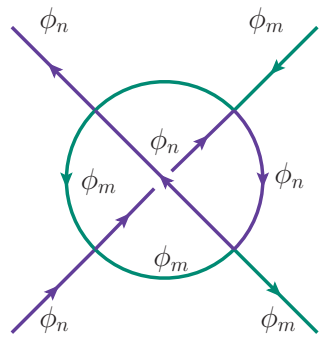

Fig 12. Every internal line is fixed to be labelled by $m$ (green line) or $n$ (purple line), and so there is no superposition of propagators for better UV behavior.

\section{$3.2 \quad$ Spinor electrodynamics}

It is not difficult to generalize this framework to fermions. The Lagrangian is of the form

$$
\begin{aligned}
\mathcal{L}= & \sum_{n} \frac{-1}{c_{n}}\left(i \bar{\Psi}_{n} \not \partial \Psi_{n}+e \bar{\Psi}_{n} \gamma^{\mu} A_{\mu} \Psi_{n}-m_{n} \bar{\Psi}_{n} \Psi_{n}\right) \\
& -\sum_{a} \frac{1}{b_{a}}\left(\frac{1}{4} F_{a}^{\mu \nu} F_{a, \mu \nu}+\frac{1}{2} M_{a}^{2} A_{a}^{\mu} A_{a, \mu}\right) .
\end{aligned}
$$

The Feynman rule is even simpler as there is a single three-point interaction for the fermion and vector.

All discussions above can be repeated easily. All results are also similar to the scalar theory. The constraints on fermion masses are slightly modified

$$
\begin{array}{lll}
\sum_{n} c_{n} m_{n}^{r}=0, & r=0,1,2,3 & \text { (from fermion self interactions), } \\
\sum_{n} e_{n}^{2} m_{n}^{r}=0, & r=0,1,2,3 \quad \text { (from fermion-vector interactions). }
\end{array}
$$

Eq.(107) is the same as (6), and (108) is the fermionic version of (84).

\subsection{Renormalization}

Massive vector field theories, as well as $\phi^{n}$ theories for $n>4$ in four dimensions, have been considered non-renormalizble. These theories require infinite number of counter terms and they contain an infinite number of free parameters.

In our theory, we also have an infinite number of parameters, but they are not totally free. To satisfy finiteness conditions such as (2), the possible choices of these parameters are restricted. Furthermore, by calculating the explicit form of an amplitude by analytic continuation, we can also compare the calculation with experimental data and determine which choice of parameters is correct.

Our recipe can also be viewed as merely a new method of regularization. After removing all UV-divergence, we can talk about how coupling constants run with energy, and we can 
still apply the renormalization procedure to our theory. It will be very interesting to study the properties of the renormalization group flow of our models.

\subsection{Future works}

An important generalization of our results is to extend it to non-abelian gauge theories. For Abelian gauge symmetry, a renormalizable, unitary perturbative theory of the massive vector boson can be constructed with the help of a Stueckelberg field. But non-Abelian gauge symmetry is much harder to deal with. Despite years of efforts, Higgs mechanism remains the only way to maintain unitarity and renormalizability for the existence of massive vector bosons. It will be very interesting to see whether our idea can be generalized to deal with non-Abelian theories, and to construct an alternative mechanism to give masses to vector bosons.

\section{Acknowledgment}

The authors thank Chuan-Tsung Chan, Jiunn-Wei Chen, Hsien-chung Kao and Yeong-Chuan Kao for helpful discussions. This work is supported in part by the National Science Council, and the National Center for Theoretical Sciences, Taiwan, R.O.C.

\section{References}

[1] Pei-Ming Ho and Yi-Ya Tian, UV-finite scalar field theory with unitarity, [hepth/0410248v2]

[2] Pei-Ming Ho and Xue-Yan Lin, A UV completion of scalar field theory in arbitrary even dimensions, Journal of High Energy Physics, Springer Berlin / Heidelberg, 10.1007/JHEP01(2010)032 pp1-15. [hep-th/0910.3287v1]

[3] R.E. Cutkosky, "Singularities And Discontinuities Of Feynman Amplitudes," J. Math. Phys. 1, 429 (1960)

[4] N. Itzhaki, Some interesting properties $f$ field theories with an infinite number of fields, Int. J. Mod. Phys. A 13, 625 (1998)

[5] Aitchison, I.J.R. , Hey, A.J.G.(1982), Gauge Theories in Particle Physics, Adam Hilger.

[6] Gastmans R. 1975 Weak and Electromagnetic Interactions at High Energies, Cargese, 1975 ed M Levy et al (New York: Plenum) pp 109ff.

[7] Cornwall, John M. and Levin, David N. and Tiktopoulos, George, Derivation of gauge invariance from high-energy unitarity bounds on the $S$ matrix, PhysRevD.10.1145, 1974 
[8] C.H. Llewellyn Smith, Phys. Letters 46B, 233, 1973

[9] M.E. Peskin, D.V. Schroeder, An Introduction to Quantum Field Theory, Addison-Wesley Advanced Book Program(now Perseus Books) (1995)

[10] Stefan Pokorsky, Gauge Field Theories, 2nd Edition, page 140-143, Cambridge Monographs on Mathematical Physics (1999) 


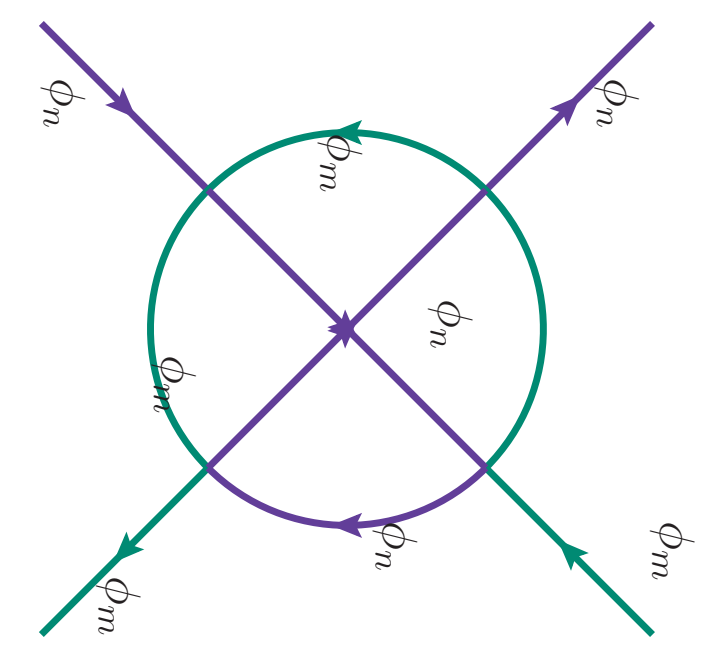

